\title{
O processo decisório do consumidor na intenção de uso de vídeo e livros eletrônicos educacionais na Internet
}

\author{
The consumer decision-making process in the intention to use the educational \\ electronic video and books on the internet
}

\author{
Rodrigo Campos Ferreira ${ }^{1 *}$, Gérson Tontini ${ }^{1}$ \\ ${ }^{1}$ Universidade Regional de Blumenau (FURB).
}

\begin{tabular}{l} 
I N F O A R T I G O \\
\hline Palavras-chave: \\
Vídeo digital educacional, \\
Livro digital educacional, \\
Comportamento do \\
consumidor.
\end{tabular}

ARTICLE INFO

Keywords:

Digital educational

video,

Digital educational

book,

Consumer

behavior.

\begin{abstract}
RESUMO
Com o intuito de entender o consumidor do mercado de produtos digitais educacionais, este estudo teve como objetivo analisar as principais características que influenciam na intenção de uso de vídeos educacionais e livros digitais educacionais na internet. Para essa análise, adaptou-se o modelo UTAUT2 (Teoria Unificada de Aceitação e Uso de Tecnologia) aos produtos digitais educacionais. A pesquisa foi realizada por meio de uma análise multivariada (fatorial exploratória e confirmatória), valendo-se de uma amostra de 460 respondentes por meio de uma pesquisa quantitativa, caracterizada como descritiva, de corte transversal e de método survey. Foram realizados testes de confiabilidade Alpha de Cronbach, Confiabilidade Composta e Variância Média Extraída e, por fim, foi realizada a Modelagem de Equações Estruturais por meio do software SmartPLS. As dimensões analisadas foram: Segurança, Influência Social, Expectativa de Desempenho, Expectativa de Esforço, Condições Facilitadoras, Motivação Hedônica, Preço, Hábito e Intenção de Uso.
\end{abstract}

\footnotetext{
* Correspondência para autor: rodrigo.camposferreira@yahoo.com (Ferreira, R.C.) (0000-0001-9758-6877), gersontontini@gmail.com (Tontini, G.) (0000-0002-7430-562X).
}

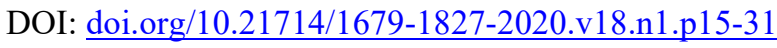

1679-1827 (C) 2019 Gest@o.org. 


\section{Introdução}

O uso da internet é cada vez maior, assim como o número de consumidores on-line, o que motiva muitos pesquisadores a desenvolverem modelos que expliquem fenômenos ligados ao comércio eletrônico (HWANG; JEONG, 2016). Acompanhar as mudanças que ocorrem no comércio on-line é um desafio no estudo do comportamento do consumidor que hoje procura entender um indivíduo ativo que se envolve em diferentes experiências virtuais de consumo, vivenciando um consumo hedonista, que cria laços com outros indivíduos e comunidades e contrasta com a teoria neoclássica de consumo voltada ao utilitarismo (TORRES, 2015).

A evolução tecnológica desencadeia uma série de transformações na sociedade que vai adaptando-se a novas formas de realizar as atividades habituais. Numa economia criativa com intenso movimento comercial na internet, novas profissões estão se consolidando e requerendo formações específicas aliadas às mídias sociais. O meio digital tem possibilitado diversas tranformações culturais e sociais que afetam os indivíduos e a maneira na qual interagem com os meios tecnológicos. Com isso surgem novos anseios por parte das pessoas, que buscam qualificações específicas que estejam alinhadas a sua realidade de compartilhamento, acesso rápido e descentralizado das informações. (GARCIA, 2015).

Cada vez mais temos um processo de aquisição de conhecimento autônomo, onde as pessoas vão construindo seu conhecimento motivadas por algo específico que desejam aprender. Essa busca criou oportunidades para que pessoas com determinadas habilidades, que desejam vender e compartilhar seu conhecimento, o façam por meio das ferramentas disponíveis na internet (LITTO, 2014). O desafio de suprir as lacunas que vão sendo criadas com a demanda crescente por produtos educacionais que se adaptem a realidade e expectativas dos alunos pode estar nos produtos digitais. Criados a partir de conteúdo adaptado do ambiente físico, ou totalmente desenvolvidos nos meios virtuais, os produtos digitais educacionais podem ser provenientes de organizações educacionais, grupos sem fins educacionais ou criados por pessoas comuns. Os produtos digitais educacionais são totalmente virtuais e podem ser acessados nos ambientes virtuais ou copiados por meio de download na internet, sendo os vídeos e livros digitais ferramentas comuns de disseminação do conhecimento (HUI; CHAU, 2002).

O presente estudo tem como objetivo analisar as principais características que influenciam na intenção de uso de um produto digital educacional. Consumidores que fazem uso de um produto digital utilizam aparatos tecnológicos para isso. Por adaptar-se bem a estas condições, o modelo UTAUT2 foi escolhido para análise do estudo. Este modelo é o resultado do aprimoramento de oito diferentes modelos de análise de aceitação da tecnologia. O UTAUT2 possui maior poder preditivo que os modelos anteriores e pode ser adaptado a diferentes construtos que possam vir a explicar a motivação do consumidor de tecnologia.

O estudo analisou o modelo UTAUT2 para produtos digitais educacionais, especificamente os produtos vídeos e livros eletrônicos com foco educacional, obtendo resultados que contribuem na consolidação do modelo utilizado e na comparação de estudos que analisaram diferentes tecnologias. Os dados obtidos também proporcionam identificar os fatores que mais influenciam o consumidor de produtos digitais educacionais, de forma que os gestores possam empregar estratégias que considerem esses resultados alinhados a seus objetivos. As organizações podem usar os diferenciais que o comércio eletrônico propicia, que sejam relevantes ao seu consumidor, de forma a aumentar a qualidade do serviço que entrega (MOREIRA, 2016).

Com o propósito de fundamentar as hipóteses da presente pesquisa, o artigo é estruturado abordando, primeiramente, aspectos do comportamento do consumidor, seguido do comércio eletrônico, posteriormente a teoria unificada da aceitação e uso da tecnologia, UTAUT2. Em seguida apresenta-se a metodologia de pesquisa utilizada, seus resultados e conclusões. Como resultado conclui-se que o Hábito, Motivação Hedônica, Expectativa de Desempenho e o Sexo, quando relacionado a Segurança, são motivadores na intenção de uso de vídeos e livros eletrônicos educacionais.

\section{Comportamento do Consumidor}

O comportamento do consumidor estuda a motivação de compra das pessoas, processo que leva os indivíduos a tomarem a decisão de gastar seus recursos para consumir algo (Howard, 1989; Schiffman; Kanuk 2000). A tomada de decisão de compra envolve uma sucessão de trocas que fazem parte da aquisição, consumo, disposição e experiências de uso de um produto ou serviço. Uma empresa só pode existir na medida 
em que compreende e satisfaz as necessidades e desejos de seus clientes (MOWEN; MINOR, 2003; SOLOMON, 2016).

Durante o processo de decisão de compra existem diferentes fatores que influenciam o consumidor para um estado de motivação no qual o indivíduo está numa condição de tensão que produz ações a fim de dissipar a tensão existente. A motivação está associada a um procedimento gerado por um estímulo que desencadeia um processo de aproximação ou distanciamento de um determinado produto, de acordo com as informações e percepções vivenciadas pelo cliente (SHETH; MITTAL; NEWMAN, 2001).

No momento que uma pessoa percebe que seu estado atual pode ser melhorado com aquisição de um produto é iniciado o processo de tomada de decisão de compra. Conforme Blackwell, Miniard e Engel (2005), este processo é compreendido por sete estágios: reconhecimento da necessidade, busca de informações, avaliação de alternativas pré-compra, compra, consumo, avaliação pós-consumo e descarte. É um processo no qual diferentes fatores relacionados com as diferenças individuais de cada pessoa, as influências ambientais e os processos psicológicos podem ocasionar mudança no comportamento e atitude do consumidor.

Para Solomon (2016) o processo de decisão de compra envolve questões pré-compra, questões do momento da compra e questões pós-compra. O foco deste estudo está na intenção de compra, que se relaciona com a fase de pré-compra, que procura responder como o consumidor decide que precisa ou deseja o produto, e as fontes de informações envolvidas nesse processo.

\section{Comércio Eletrônico}

O comércio eletrônico, ou e-commerce, é uma opção de varejo por meio da internet onde pessoas, físicas ou jurídicas, realizam algum tipo de transação comercial. Ganhou espaço no comércio especialmente pela sua facilidade de troca de informações e atualmente é o modelo de negociação que mais cresce no mercado. Existem características distintas no comércio eletrônico como seu alcance global e facilidade de acesso em diferentes dispositivos eletrônicos. O consumidor digital se difere do consumidor tradicional por vivenciar uma experiência de estar imerso num ambiente digital que é complexo, sensível e perceptual (GOMES et al., 2017).

O comércio eletrônico oportuniza ao consumidor fornecer as especificações e informações relativas ao pedido feito, maximizando a precisão por parte do fornecedor na entrega do melhor produto (MOREIRA, 2016). A medida que o consumidor interage e conhece os produtos e serviços das empresas, ele vai se tornando mais exigente e participativo (GOMES et al., 2017). A manutenção de relacionamentos lucrativos e duradouros requer das empresas que atuam com comércio eletrônico a análise dos aspectos relevantes para criar uma percepção de alto nível de qualidade diante dos consumidores. Isso demanda uma busca constante por entender as necessidades e desejos do cliente a fim de entregar da melhor forma o produto certo (MACEDO et al., 2013).

O consumidor virtual busca satisfação comprando determinados produtos que expressam um valor desejado específico, variando de acordo com as preferências, possibilidades e aspirações próprias. Quando isso ocorre, ele espera que o processo de compra ocorra da forma mais simples e rápida possível (MACEDO et al., 2013; JACIOW; WOLNY; STOLECKA-MAKOWSKA, 2013). O comportamento de compra também é moldado pelas tendências de consumo que podem existir por um curto ou longo período e ter abrangência regional ou global (JACIOW; WOLNY; STOLECKA-MAKOWSKA, 2013).

O ambiente virtual possibilitou o desenvolvimento de produtos digitais em diversos segmentos. São bens ou serviços que podem ser digitalizados e consumidos no ambiente virtual como os softwares, músicas, vídeos, fotos, livros e outros (HUI; CHAU, 2002). Assim, como o conhecimento está sendo disseminado por meio dos ambientes virtuais, a educação também tem, a cada dia mais, se inserido no mundo digital. A arquitetura do produto digital educacional é dividida em estrutura e conteúdo. O conteúdo pode ser alocado em diferentes estruturas lógicas ou físicas. No caso do e-book, o conteúdo pode ser representado no formato de texto. $\mathrm{O}$ vídeo digital tem seu conteúdo expresso por imagens dinâmicas e sons. $\mathrm{O}$ acesso a esses produtos ocorre em diferentes plataformas por meio da internet. Essas plataformas são baseadas em banco de dados para o armazenamento e recuperação do conteúdo (SORDI et al., 2016). 
O ensino no meio eletrônico, também conhecido como e-learning, é um modelo de ensino a distância suportado por tecnologia que integra grande variedade de materiais de instrução como texto, áudio ou vídeo. Pode permitir a interação entre aluno e professor ou entre os próprios alunos por meio de salas de bate papo, e-mail, fóruns e outros (CHANG; HAJIYEV; SU, 2017). O e-learning está presente como alternativa aos métodos tradicionais de ensino. As novas gerações de estudantes já estão habituadas a interagir no ambiente virtual pesquisando na internet as informações e conteúdos que são de seu interesse. Esses indivíduos certamente terão dificuldade em aceitar e se adaptar ao modelo de ensino baseado em aulas expositivas que fornecem respostas aos alunos sem que eles tenham despertado qualquer curiosidade a respeito delas (TORI, 2015).

Os vídeos e livros eletrônicos educacionais são opções de aprendizagem naturais para usuários da internet que buscam por informações. A importância do vídeo na vida das pessoas pode ser percebida nas redes sociais virtuais baseadas puramente em vídeos. Os ambientes de aprendizagem se ampliaram, e os meios de ensino devem estar adaptados aos sujeitos que participam do processo de aprendizagem e acessam o conteúdo em diferentes períodos e lugares (LUNA, 2014). Os vídeos digitais e recursos multimídias educativos em geral permitem aos alunos controlar seu próprio ritmo de aprendizado, respeitando as diferenças individuais no aprendizado (KARPPINEN, 2005).

O surgimento dos livros eletrônicos trouxe consigo a possibilidade de proporcionar novas experiências de leitura, interatividade e portabilidade às pessoas (ALMEIDA; NICOLAU, 2013). Empresas de ecommerce, que comercializam e-books, podem implementar ferramentas que melhoram a experiência de compra dos consumidores. Essas ferramentas fornecem indicações de livros de acordo com as informações que possuem do indivíduo, também é possível cruzar informações com o comportamento de compra de outras pessoas que adquiriram produtos similares. Ao buscar algum tipo de conhecimento na aquisição de um ebook, o consumidor pode ser levado a conhecer outros livros digitais afins, assim como outros assuntos de interesse, despertando a curiosidade e a busca pelo conhecimento (MARASSI, 2013).

Durante o processo de compra de vídeos e livros eletrônicos educacionais os consumidores podem utilizar as informações existentes na internet para compor suas decisões de compra, minimizando riscos e incertezas. A percepção do risco pode ser entendida como a expectativa de perda após a realização de uma compra. Para que uma compra seja efetivada, é preciso que os consumidores sintam segurança na loja virtual em que pretendem comprar (BEHRENS, 2014). Não oferecer procedimentos e ferramentas certificados e atualizados pode custar o sucesso do comércio virtual. O sentimento de insegurança é um fator que poderá inviabilizar a compra (MORGADO, 2003; MACEDO et al., 2013; FERREIRA, 2013; GOMES et al., 2017). Com base na importância da segurança no processo de compra on-line, foi formulada a seguinte hipótese:

H1: A sensação de segurança influencia positivamente na intenção de compra do produto digital educacional.

Ao considerar a relevância que as percepções dos indivíduos têm na decisão de compra, Ferreira (2013) realizou um estudo considerando a diferenciação quanto ao comportamento de compra on-line entre o sexo masculino e o feminino, encontrando diferenças especialmente no que tange ao caráter emocional, de conveniência e de motivações utilitárias. Os resultados indicam que os homens têm comportamento mais impulsivo nas compras on-line, tendo tendência a gastar mais nas compras que realizam.

Outros estudos também analisaram as diferenças de comportamento de compra na internet entre homens e mulheres, como Casimir e Dutilh (2003), Eagly et al. (2004), Luchs e Mooradian (2012) e Jones et al. (2017) que concluiram que as mulheres têm maior preocupação com questões relativas ao impacto ambiental e social do consumo. Já os estudos de Riquelme e Rios (2010), Gutiérrez, López-Catalán e Ramon-Jeronimo (2012), Okazaki e Mendez (2013) e Ames et al. (2016) identificaram diferenças significativas em apenas algumas variáveis que correspondem a percepção entre homens e mulheres que realizam compras on-line por meio de dispositivos móveis no chamado m-commerce. Considerando as diferenças encontradas entre o sexo masculino e o feminino, surge a seguinte hipótese:

H2: Existe diferença significativa no comportamento de compra de produtos digitais educacionais entre homens e mulheres.

As hipóteses restantes que compõe a análise deste trabalho estão relacionadas aos construtos que 
fazem parte do modelo da Teoria Unificada da Aceitação e Uso de Tecnologia e serão abordadas de forma agrupada após a apresentação do modelo e seus respectivos construtos.

\section{Teoria unificada da aceitação e uso de tecnologia}

Estudos que avaliam o uso e adoção de tecnologias incorporadas ao comércio eletrônico consideram que o consumidor tem que interagir com a tecnologia para realizar uma compra on-line (HWANG; JEONG, 2016). O entendimento do comportamento do usuário de tecnologia da informação passou por diferentes estudos. Segundo Venkatesh et al. (2003), as pesquisas anteriores nessa área têm origens nas áreas de sistema de informação, psicologia e sociologia. A escolha por um modelo de análise de intenção de uso de tecnologia é difícil devido ao grande número de modelos existentes que sozinhos não respondem mais do que $40 \%$ da variação no comportamento de uso tecnológico.

A UTAUT é resultado do trabalho de Venkatesh et al. (2003), que unificaram oito modelos de análise da aceitação da tecnologia:

1.Teoria da Ação Racional (TRA) de Fishbein e Ajzen (1975) - defende a ideia de o comportamento individual ser regido de acordo com atitudes relacionadas a sentimentos positivos e negativos.

2.Modelo de Aceitação da Tecnologia (TAM) de Davis (1989) - analisa as atitudes para usar o Sistema de Informação (SI), definindo um comportamento de utilização da tecnologia.

3.Modelo Motivacional (MM) de Vallerand (1997) - com base em construtos de motivação intrínsecas e extrínsecas, utiliza teorias motivacionais para explicar o comportamento dos indivíduos.

4.Teoria do Comportamento Planejado (TPB) de Ajzen (1991) - partindo da TRA, acrescenta o construto de controle do comportamento para determinação da intenção e comportamento do uso da tecnologia.

5.Modelo combinado TAM-TPB de Taylor e Tood (1995) - incluí no modelo híbrido TAM-TPB os construtos atitude para o comportamento, normas subjetivas, controle comportamental percebido e utilidade percebida.

6.Modelo de Utilização do PC (MPCU) de Thompson et al. (1991) - analisa os efeitos de construtos como ajuste ao trabalho, complexidade, consequências de longo prazo, efeitos em razão do uso, fatores sociais e condições facilitadoras na intenção de uso dos PCs (Personal Computers).

7.Teoria da Difusão da Inovação de Rogers (1995) e Moore e Benbasat (1996) - contempla características de inovação com construtos como vantagem relativa, facilidade de uso, imagem, visibilidade, compatibilidade, demonstração de resultado e uso voluntário para o estudo de aceitação individual da tecnologia.

8. Teoria Social Cognitiva de Bandura (1986) e ampliada para o uso de computadores por Compeau e Higginst (1995) - utiliza construtos como a expectativa de resultados de performance pessoal, autoeficácia, afeto e ansiedade no estudo do uso dos computadores. Esse modelo possibilita que sejam analisadas a aceitação e o uso de tecnologias da informação.

A UTAUT foi desenvolvida com foco na adoção de tecnologia para usuários de organizações, não abrangendo quem adquiria a tecnologia, mas sim quem a utilizava num contexto laboral. 
Figura 1 - Modelo UTAUT

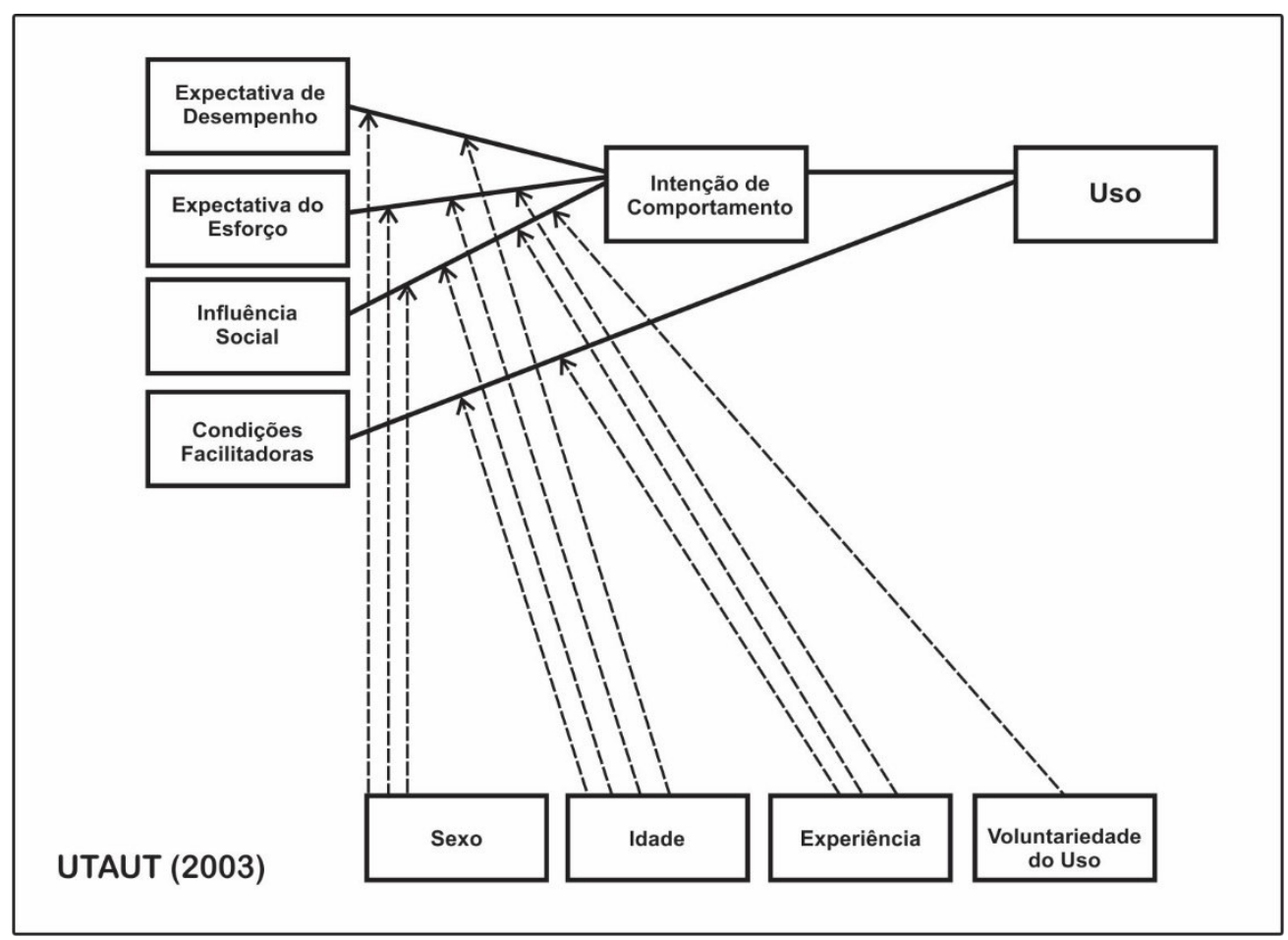

Fonte: Venkatesh et al. (2003)

Com o intuito de expandir seu uso, Venkatesh, Thong e Xu (2012) desenvolveram a UTAUT2, relacionando a intenção de uso com o comportamento do consumidor de tecnologia. Para aprofundar a análise, para indivíduos que além de usar a tecnologia também a compram, esse novo modelo incluiu novos construtos: motivação hedônica, preço e hábito. Também foi excluído do construto o uso voluntário, por não se relacionar a comportamentos de compra.

Figura 2 - Modelo UTAUT2

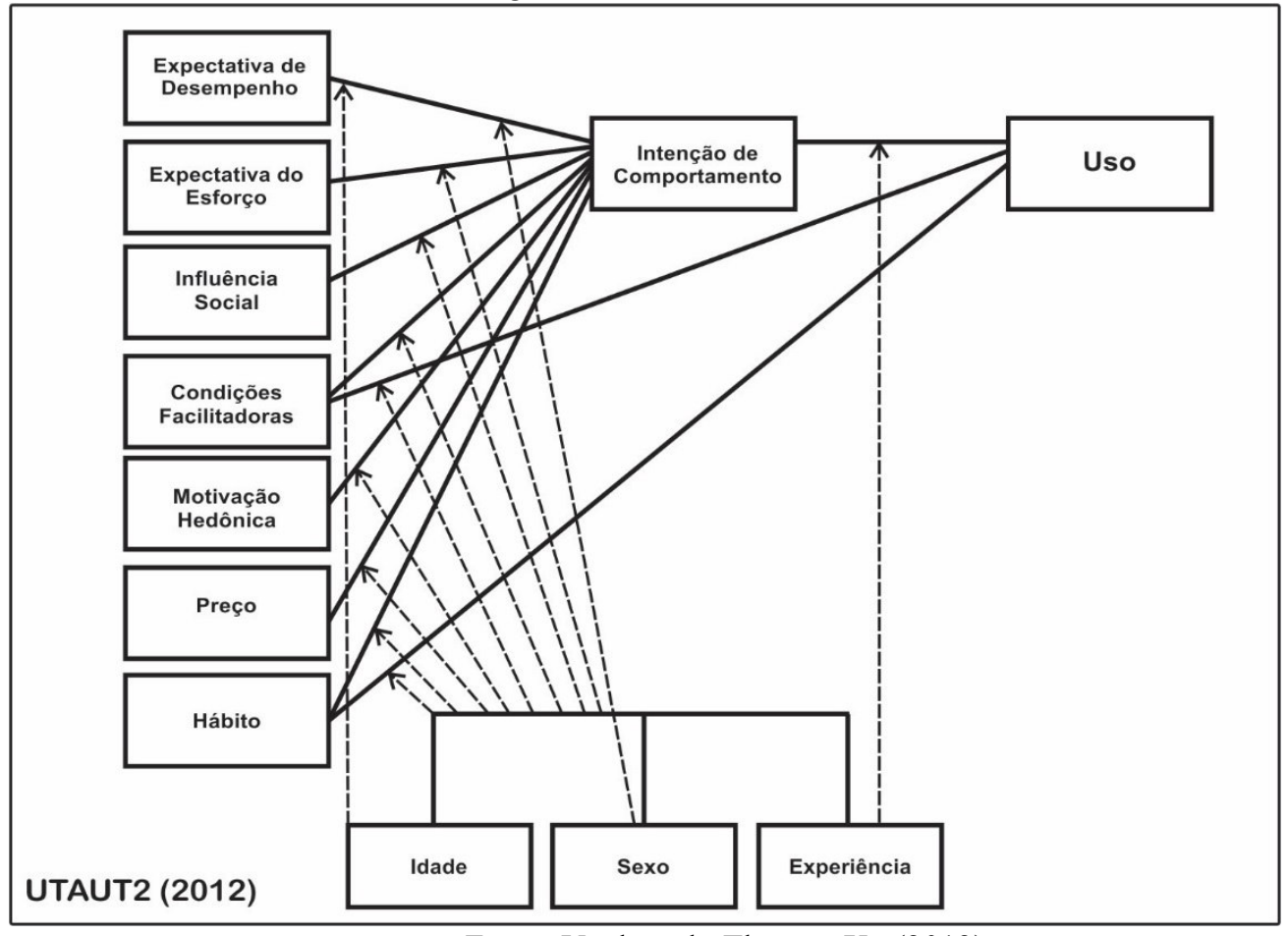

Fonte: Venkatesh, Thong e Xu (2012) 
Os sete fatores presentes no modelo UTAUT2 relacionados a intenção de comportamento de compra são apresentados no Quadro 1.

Quadro 1 - Fatores do modelo UTAUT2

\begin{tabular}{|c|c|}
\hline Fatores & Construtos \\
\hline Expectativa de desempenho & $\begin{array}{l}\text { Quanto o consumidor acredita que o uso do produto o ajudará a alcançar o } \\
\text { benefício almejado. }\end{array}$ \\
\hline Expectativa de esforço & $\begin{array}{l}\text { Quanto o consumidor acredita que irá dispender de esforço para utilizar o } \\
\text { produto. }\end{array}$ \\
\hline Influência social & $\begin{array}{l}\text { Quanto o consumidor percebe de importância da opinião dos outros na } \\
\text { avaliação do produto. }\end{array}$ \\
\hline Condições facilitadoras & $\begin{array}{l}\text { Quanto o consumidor acredita na infraestrutura organizacional e técnica } \\
\text { que dá suporte ao uso do produto. }\end{array}$ \\
\hline Motivação hedônica & $\begin{array}{l}\text { Quanto o consumidor percebe o uso do produto como sendo divertido ou } \\
\text { prazeroso. }\end{array}$ \\
\hline Preço & Quanto o valor monetário irá influenciar na adoção do produto. \\
\hline Hábito & $\begin{array}{l}\text { Quanto o consumidor já teve de experiência com elementos correlatos ao } \\
\text { do produto. }\end{array}$ \\
\hline Intenção de comportamento & $\begin{array}{l}\text { De que forma o consumidor irá se comportar, se pretende utilizar } \\
\text { determinado produto. }\end{array}$ \\
\hline
\end{tabular}

Fonte: Desenvolvido pelo autor

De acordo com os fatores do modelo UTAUT2 seguem as hipóteses restantes formuladas para este estudo:

- H3: A expectativa de desempenho influencia positivamente a intenção de uso do produto digital educacional.

- H4: A expectativa de esforço influencia positivamente a intenção de uso do produto digital educacional.

- H5: A influência social influência positivamente a intenção de uso de um produto digital educacional.

- H6: As condições facilitadoras influenciam positivamente a intenção de uso de um produto digital educacional.

- H7: A motivação hedônica influencia positivamente a intenção de uso de um produto digital educacional.

- H8: O preço influencia positivamente a intenção de uso de um produto digital educacional.

- H9: O hábito influencia positivamente a intenção de uso de um produto digital educacional. 


\section{Procedimentos Metodológicos}

Para atingir o objetivo deste estudo, que é analisar as principais características que influenciam a intenção de uso de um produto digital educacional, foi realizada uma análise multivariada fatorial exploratória e confirmatória. A análise multivariada possibilita a identificação das ligações existentes nas variáveis envolvidas no objeto de estudo. Mensurar diversas variáveis simultaneamente auxilia de forma específica e precisa na formulação de questões de relativa complexidade. (HAIR JR et al., 2005).

Foi realizada uma pesquisa descritiva, a qual se propõe a mensurar as diferentes características apontadas numa questão de pesquisa. (HAIR JR et al., 2005). O questionário foi elaborado tendo como base os construtos e assertivas do modelo UTAUT2 adaptados aos produtos digitais educacionais (Anexo A). São elementos de pesquisa que já foram validados anteriormente por diferentes estudos, em especial os estudos de Venkatesh et al. (2003) e Venkatesh, Thong e Xu (2012), que deram origem ao modelo. A escala de respostas utilizada foi a de Likert, de cinco pontos, escala psicométrica mais utilizada em questionários e pesquisas de opinião.

Antes de iniciar a coleta dos dados utilizados na pesquisa foi realizado um pré-teste com uma turma do curso de graduação em administração. Posteriormente, algumas alterações foram realizadas nas questões em relação a redação das perguntas. Para coleta de dados utilizou-se um questionário autoaplicado que foi disponibilizado por 15 dias por meio de um formulário on-line da ferramenta Google Docs. O contato com os respondentes ocorreu por meio das redes sociais digitais e também por e-mail.

A pesquisa foi de corte transversal, caracterizada pela coleta de informações uma única vez em determinado período de tempo (MALHOTRA, 2001). O método utilizado foi o survey, ou levantamento, que envolve a interrogação direta de um determinado grupo significativo de pessoas, as quais possuem um comportamento que se deseja conhecer. A utilização do survey é comum em amostras consideradas grandes (HAIR JR et al., 2005). Como a aquisição e o consumo de um produto digital acontece totalmente por meio da internet, os critérios adotados para escolha da amostra foram o uso da internet e a existência de alguma experiência de aprendizado com o uso de vídeos e livros eletrônicos.

Devido a amostra ser caracterizada apenas por indivíduos que já apresentam experiência no uso de vídeos e livros eletrônicos optou-se por descartar a análise da variável moderadora experiência, constante no modelo UTAUT2. Os critérios abrangem praticamente qualquer usuário da internet, visto que o acesso facilitado a vídeos e textos instrucionais como tutoriais e diferentes formatos de livros eletrônicos é algo comum na rotina desses usuários. Considerando o universo dos usuários da internet, os participantes da amostra foram formados com base na sua própria disposição e conveniência para participar da pesquisa (HAIR JR et al., 2005), o que caracteriza a amostragem não probabilística por conveniência (MALHOTRA, 2001). O número de respondentes desta pesquisa foi de 460 indivíduos.

A avaliação do modelo foi realizada verificando o Alpha de Cronbach (A.C.), a consistência interna por meio da Composite Reability (C.R.), seguida da validade convergente analisada por meio do Average Variance Extracted (A.V.E.) e da validade discriminante estimada pelo critério de cargas cruzadas (HENSELER; RINGLE; SINKOVICS, 2009). Os dados coletados foram organizados e analisados de forma descritiva por meio do software SmartPLS, versão 3.2.7. Com a finalidade de analisar as características mais relevantes na intenção de uso de um produto digital educacional por meio das variáveis estudadas, realizou-se o estudo por intermédio de análise de equações estruturais.

\section{Apresentação e Discussão dos Resultados}

Com os resultados da pesquisa disponíveis a análise dos dados foi feita inicialmente com a caracterização das pessoas que responderam ao questionário. Esta etapa ocorreu por meio da observação da frequência das respostas que proporcionou a construção do perfil dos respondentes da pesquisa. Reunindo as principais características dos respondentes foi possível determinar um perfil genérico que apresentou as seguintes características: sexo feminino, solteira, idade entre 30 e 39 anos, residente na Região Sul do Brasil e possui renda familiar entre $\mathrm{R} \$ 3.748,01$ e $\mathrm{R} \$ 9.370,00$.

Posteriormente foi analisada a confiabilidade individual, que trata das correlações existentes nas assertivas do questionário. O seu grau de correlação expressa o poder explicativo de determinada variável. 
Conforme a Tabela 2 é possível verificar que os testes Average Variance Extracted com valor $\geq$ a 0,5 (FORNELL; LACKER, 1981) e Alpha de Cronbach $\geq$ a 0,6 (HILL; HILL, 2000), obtiveram valores dentro dos padrões adequados. Similar ao Alpha de Cronbach, o Composite Reability é um indicador de confiabilidade. Esse coeficiente também obteve resultados acima do valor aceitável $\geq$ a 0,7 (CHIN, 1998) em todos os construtos.

Tabela 2 - Testes de confiabilidade

\begin{tabular}{l|c|c|c}
\hline Construtos & A.C. & C.R. & A.V.E. \\
\hline 01 - Segurança & 0,750 & 0,853 & 0,664 \\
\hline 02 - Influência Social & 0,767 & 0,850 & 0,587 \\
\hline 03 - Expectativa de Desempenho & 0,854 & 0,901 & 0,695 \\
\hline 04 - Expectativa de Esforço & 0,791 & 0,856 & 0,548 \\
\hline 05 - Condições Facilitadoras & 0,631 & 0,795 & 0,568 \\
\hline 06 - Motivação Hedônica & 0,822 & 0,882 & 0,652 \\
\hline 07 - Preço & 0,855 & 0,912 & 0,775 \\
\hline 08 - Hábito & 0,731 & 0,848 & 0,651 \\
\hline 09 - Intenção de Uso & 0,894 & 0,927 & 0,761 \\
\hline
\end{tabular}

Fonte: Dados da pesquisa

A validade discriminante avalia um conjunto de indicadores a fim de verificar se eles se diferenciam um do outro. São avaliadas a matriz de correlação estimada e a raiz quadrada de variância média extraída dos construtos. Os dados da validade discriminante obtidos neste estudo (Tabela 2) suportam o critério de Fornell e Larcker (1981), no qual o valor de cada coluna é maior do que aqueles que estão abaixo e representam a correlação entre os construtos (RINGLE; DA SILVA; BIDO, 2014).

Tabela 3 - Validade discriminante

\begin{tabular}{|c|c|c|c|c|c|c|c|c|c|}
\hline & 01 - SEG & 02 - IS & 03 - ED & $04-E E$ & $05-C F$ & 06 - МН & 07 - PRE & 08 - НАВ & 09 - IU \\
\hline 01 - Segurança & 0,815 & & & & & & & & \\
\hline $\begin{array}{l}02 \text { - Influência } \\
\text { Social }\end{array}$ & 0,182 & 0,766 & & & & & & & \\
\hline $\begin{array}{l}03 \text { - Expectativa } \\
\text { de Desempenho }\end{array}$ & 0,175 & 0,255 & 0,833 & & & & & & \\
\hline $\begin{array}{l}04 \text { - Expectativa } \\
\text { de Esforço }\end{array}$ & 0,174 & 0,193 & 0,402 & 0,740 & & & & & \\
\hline $\begin{array}{l}05 \text { - Condições } \\
\text { facilitadoras }\end{array}$ & 0,211 & 0,221 & 0,228 & 0,497 & 0,753 & & & & \\
\hline $\begin{array}{l}06 \text { - Motivação } \\
\text { Hedônica }\end{array}$ & 0,161 & 0,263 & 0,412 & 0,493 & 0,250 & 0,808 & & & \\
\hline 07 - Preço & 0,157 & 0,267 & 0,271 & 0,224 & 0,221 & 0,334 & $\mathbf{0 , 8 8 0}$ & & \\
\hline 08 - Hábito & 0,103 & 0,190 & 0,499 & 0,404 & 0,216 & 0,541 & 0,227 & $\mathbf{0 , 8 0 7}$ & \\
\hline $\begin{array}{l}09 \text { - Intenção de } \\
\text { Uso }\end{array}$ & 0,121 & 0,214 & 0,509 & 0,362 & 0,177 & 0,606 & 0,292 & 0,720 & 0,873 \\
\hline
\end{tabular}

Fonte: Dados da pesquisa 
Para identificar as relações entre os construtos que representam as hipóteses consideradas neste estudo, foi realizada análise pela técnica de bootstraping para verificar os valores do teste t de Student. As hipóteses aceitas utilizando o teste $\mathrm{t}$ de Student e $p$-value tiveram como valores de referência $\mathrm{t} \geq 1,96$ e $p$-value $<0,5$ (HAIR JR et al., 2014).

Tabela 4 - Teste $T$ de Student e p-value entre os construtos

\begin{tabular}{|l|l|l}
\hline Construtos & Teste T de Student & P-value \\
\hline $\mathbf{1}$ - Segurança $\rightarrow$ Intenção de Uso & 1,036 & 0,301 \\
\hline $\mathbf{2}$ - Influência Social $\rightarrow$ Intenção de Uso & 0,005 & 0,996 \\
\hline $\mathbf{3}$ - Expectativa de Desempenho $\rightarrow$ Intenção de Uso & $\underline{\mathbf{3 , 6 5 9}}$ & 0,000 \\
\hline $\mathbf{4}$ - Expectativa de Esforço $\rightarrow$ Intenção de Uso & 1,389 & 0,165 \\
\hline $\mathbf{5}$ - Condições Facilitadoras $\rightarrow$ Intenção de Uso & 0,416 & 0,678 \\
\hline $\mathbf{6}$ - Motivação Hedônica $\rightarrow$ Intenção de Uso & $\underline{\mathbf{6 , 5 2 7}}$ & 0,000 \\
\hline $\mathbf{7}$ - Preço $\rightarrow$ Intenção de Uso & 1,361 & 0,174 \\
\hline $\mathbf{8}$ - Hábito $\rightarrow$ Intenção de Uso & $\underline{\mathbf{1 1 , 9 1 6}}$ & 0,000 \\
\hline $\mathbf{1 0}$ - Sexo $\rightarrow$ Intenção de Uso & 0,579 & 0,563 \\
\hline $\mathbf{1 1}$ - Idade $\rightarrow$ Intenção de Uso & 1,912 & 0,057 \\
\hline
\end{tabular}

Fonte: Dados da pesquisa

Na sequência, é apresentado um esquema (Figura 3) que retrata o modelo completo utilizado neste estudo com as conexões referentes às hipóteses, assim como os valores obtidos no teste $t$ de Student.

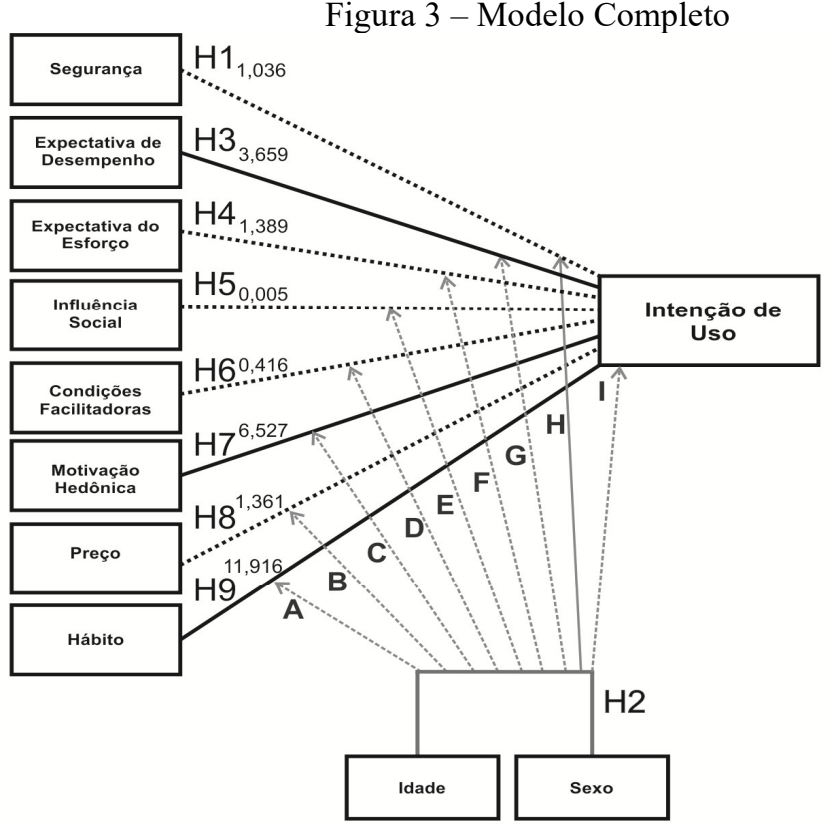

Fonte: Desenvolvido pelo autor

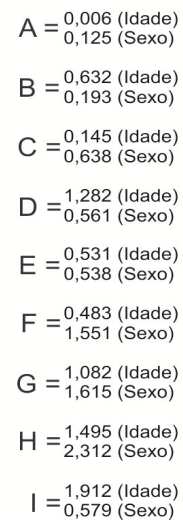


As relações entre os construtos que representam as hipóteses possuem um valor de coeficiente de caminho $(\Gamma)$, que tem o intuito de avaliar as relações causais de uma variável independente sobre uma variável dependente (RINGLE; DA SILVA; BIDO, 2014). De acordo com os resultados obtidos, o construto Hábito (relativo à experiência e frequência de uso do produto) com valor de $\Gamma=0,498$ foi o que obteve maior influência na intenção de aquisição de produtos digitais educacionais. Seguido da Motivação Hedônica (percepção de prazer ou diversão num produto) com valor de $\Gamma=0,292$, da Expectativa de Desempenho (relação do uso do produto e benefício almejado) com valor de $\Gamma=0,163$ e do Sexo quando relacionado à Segurança com valor de $\Gamma=0,019$. Conforme o valor de $\mathrm{R}^{2}$ ajustado obtido neste estudo, os construtos explicam $62,4 \%$ de sua variância.

As nove hipóteses analisadas neste estudo estão representadas no quadro abaixo junto com seus respectivos resultados:

Quadro 2 - Resultado das hipóteses

\begin{tabular}{|l|l|l|}
\hline Hipótese & Construto & Situação \\
\hline H1 & Segurança $\rightarrow$ Intenção de Uso & Não suportada \\
\hline H2 & Sexo $\rightarrow$ Intenção de Uso & $\begin{array}{l}\text { Parcialmente } \\
\text { suportada }\end{array}$ \\
\hline H3 & Expectativa de Desempenho $\rightarrow$ Intenção de Uso & Suportada \\
\hline H4 & Expectativa de Esforço $\rightarrow$ Intenção de Uso & Não suportada \\
\hline H5 & Influência Social $\rightarrow$ Intenção de Uso & Não suportada \\
\hline H6 & Condições facilitadoras $\rightarrow$ Intenção de Uso & Não suportada \\
\hline H7 & Motivação Hedônica $\rightarrow$ Intenção de Uso & Suportada \\
\hline H8 & Preço $\rightarrow$ Intenção de Uso & Não suportada \\
\hline H9 & Hábito $\rightarrow$ Intenção de Uso & Suportada \\
\hline
\end{tabular}

Fonte: Dados da pesquisa

O construto Segurança não obteve valores significativos ( $\mathrm{t}$ de Student $=1,036$ e $p$-value $=0,301)$ que justificassem sua influência na intenção de uso de produtos digitais educacionais, exceto quando relacionado a diferenças entre o sexo masculino e o feminino (pessoas do sexo feminino apresentam maior preocupação com a segurança em compras on-line). Resultados de outros estudos também indicaram pouca divergência ao analisarem possíveis diferenças no comportamento de compra em relação ao sexo dos indivíduos, como os resultados alcançados por Ames et al. (2016) e Xu (2014), que compararam o comportamento de compra de homens e mulheres no uso de dispositivos móveis para compras on-line.

No estudo de Ferreira (2013), a respeito da análise das percepções de compra do consumidor, os resultados indicaram algumas diferenças na percepção de homens e mulheres em relação às compras on-line. As mulheres apresentam maior envolvimento com as compras e menor disposição de gastos, e os homens demonstram ser mais impulsivos e com propensão a gastar mais dinheiro nas compras. Esses resultados complementam os achados neste estudo, as pessoas do sexo masculino tendo maior impulsividade tendem a se preocupar menos com a segurança nas compras on-line.

Numa pesquisa feita com clientes de telefonia móvel da Região Sudeste do Brasil, a respeito da intenção de uso do Mobile Payment, a Expectativa de Desempenho foi o construto de maior influência (ABRAHÃO, 2015). O construto também está presente na aceitação de idosos no uso das TIC. O público respondente foi de 
indivíduos com idade média de 67 anos, residentes de uma grande cidade de Portugal (MACEDO, 2017). Os resultados encontrados indicam que a intenção de compra de um produto digital educacional poderá se efetivar de acordo com a percepção que o indivíduo tem do quanto o vídeo ou livro digital educacional irá proporcionar de benefício após ter sido adquirido.

A Motivação Hedônica, relacionada à percepção de uso do produto, sendo divertido ou prazeroso, obteve resultados significativos, confirmando sua influência na intenção de uso de produtos digitais educacionais. Também demonstrou ser relevante no estudo de Silveira (2012), que indicou que a Motivação Hedônica impacta na adoção da internet móvel em jovens adultos, mesmo resultado obtido por Alalwan et al. (2017) ao analisar os fatores que influenciam na intenção e adoção de internet banking para consumidores jordanianos. A Motivação Hedônica foi o construto de maior influência quando analisada a aceitação de $e$ readers, aparelhos projetados para facilitar a leitura digital, em especial a leitura de e-books (ANDRADE; RAMOS; PEREIRA, 2013).

O construto Hábito obteve valores que confirmam sua influência na intenção de uso de produtos digitais educacionais. Da mesma forma que um estudo realizado com o consumidor português que encontrou evidências que indicam a influência desse construto na aceitação de dispositivos wearables - aparelhos eletrônicos portáteis que podem ser integrados a roupas e adereços e visam a aprimorar as capacidades humanas (ALVES, 2016). O Hábito também obteve resultados significativos no estudo relacionado à intenção de uso de sites de compras coletivas, sendo o construto de maior poder preditivo (MORAES; CAPPELLOZZA; MEIRELLES, 2017).

O construto Hábito se relaciona à execução de comportamentos automáticos resultantes da aprendizagem e experiência do indivíduo (LIMAYEM; HIRT; CHEUNG, 2007). São comportamentos presentes no uso dos vídeos e livros eletrônicos da maior parte dos usuários da internet. Isso reflete a importância que o uso desses produtos seja integrado de forma harmônica aos métodos de acesso já existentes na internet, evitando qualquer desconforto e necessidade de aprendizagem por parte do usuário.

Conforme os resultados obtidos, os demais construtos que não obtiveram resultados significativos, como a Segurança, Expectativa de Esforço, Influência Social, Condições Facilitadoras e Preço, correspondem a atributos que não exercem influência na intenção de aquisição e uso de um produto digital educacional. Mas é importante que correspondam aos parâmetros já existentes na internet, pois fazem parte da experiência que o consumidor acredita previamente que seja suprida sem que isso agregue qualquer diferencial ao produto.

São os atributos salientes e importantes de Alpert (1971) ou básicos e discriminadores de McMillan e McGrath (1996). Classificações que representam atributos que não possuem importância no processo de decisão de compra ou aqueles que os consumidores já esperam encontrar nos produtos. Solomon (2016) orienta que, quando necessário, se crie estratégias de comunicação que destaquem estes atributos em contraste com os outros para serem percebidos, de acordo com os objetivos organizacionais.

\section{Considerações Finais}

As contribuições teóricas do presente estudo estão relacionadas aos objetivos propostos. Inicialmente o de identificar os principais fatores que motivam a intenção de uso de um produto digital educacional. Em relação a esse objetivo foi possível constatar, de acordo com as hipóteses aceitas, os construtos Hábito, Motivação Hedônica, Expectativa de Desempenho e o Sexo, quando relacionado a Segurança, como motivadores na intenção de uso de vídeos e livros eletrônicos educacionais. O modelo foi validado utilizando testes de confiabilidade e validade discriminante, conforme apresentado nos procedimentos metodológicos, obtendo um poder preditivo de $62,4 \%$.

O presente estudo contribuiu ainda com a ampliação dos estudos envolvendo o modelo UTAUT2. Foram avaliadas as escalas propostas originalmente incluindo o construto Segurança. Também foi avaliada uma tecnologia diferente da internet móvel analisada no modelo original. Os vídeos e livros eletrônicos são os principais formatos de difusão do conhecimento on-line o que possibilita uma abrangente interseção de estudos. Os resultados obtidos nesta pesquisa poderão auxiliar diferentes pesquisas que abordem o tema educação por meio da internet.

Os resultados alcançados também apresentam contribuições gerenciais com os construtos que demonstraram ter influência na intenção de uso de vídeos e livros eletrônicos educacionais. Os gestores 
podem concentrar esforços nos quesitos relacionados aos construtos que motivam a aquisição e uso do produto digital educacional. $\mathrm{Na}$ Expectativa de Desempenho destacando e alinhando o benefício almejado pelo consumidor com o real benefício alcançado pelo produto. Na Motivação Hedônica promovendo e ampliando o prazer proporcionado no uso do produto. No Hábito associando os elementos que compõe o produto e seu uso a possíveis experiências correlatas do cliente. Para os fatores que não impactam a decisão de aquisição do produto, é preciso manter os padrões de qualidade, pois isso é o esperado pelo consumidor.

Outra implicação gerencial está na definição de uma persona, que é uma representação do comportamento do indivíduo diante da sociedade. Com base nas informações obtidas com a caracterização do consumidor de vídeos e livros eletrônicos educacionais, é possível criar uma persona que simbolize um coletivo por meio da representação de um indivíduo (JUNG, 2000). Os gestores que tem personas caracterizadas por pessoas do sexo feminino, ou que tem seu produto direcionado predominantemente para este público, podem incrementar seus esforços de vendas e comunicação considerando a preocupação com segurança em compras on-line apontada no fator Sexo quando associado a Segurança.

Dentre as limitações deste estudo está a concentração de respondentes na Região Sul do país, o que pode inviabilizar uma generalização quando adaptada em regiões que obtiveram baixo índice de participação na pesquisa. Futuros estudos poderão utilizar amostras melhor distribuídas pelo Brasil ou realizar pesquisas em diferentes localidades. Também poderão ampliar o estudo indo além da intenção de uso e incluir o construto uso e a experiência na moderação para mensurar o comportamento de consumidores com base no modelo original da UTAUT2. Além de incluir diferentes produtos digitais educacionais na pesquisa, assim como ferramentas utilizadas no processo de ensino on-line ou ambientes virtuais de aprendizagem específicos.

Outros trabalhos poderão explorar a definição do perfil do consumidor de produtos digitais educacionais aprofundando sua análise com a realização de uma pesquisa qualitativa. Com dados mais individualizados é possível produzir uma, ou mais, personas representativas desse público, tornando as ações e estratégias gerenciais mais assertivas.

\section{REFERÊNCIAS}

ABRAHÃO, R. de S. Intenção de adoção do mobile payment: uma análise à luz das teorias de aceitação e uso de tecnologia. 2015. 107 f. Dissertação (Mestrado em Administração) - Universidade Federal de Uberlândia, Uberlândia, 2015.

ALMEIDA, F.; NICOLAU, M. A reconfiguração do livro didático em versão digital: uma ideia de sustentabilidade. Temática, v. 9, n. 1, 2013.

ALALWAN, A. A. et al. Examining factors influencing Jordanian customers' intentions and adoption of internet banking: extending UTAUT2 with risk. Journal of Retailing and Consumer Services, v. 40, p. 125 $138,2017$.

ALPERT, M. I. Identification of determinant attributes: a comparison of methods. Journal of Marketing Research, v. 8, n. 2, p. 184-191, 1971.

ALVES, A. S. G. Um estudo empírico sobre a aceitação de dispositivos wearable pelo consumidor português. 2016. 57 f. Dissertação (Mestrado em Administração) - Universidade de Lisboa, Lisboa, 2016.

ANDRADE, A.; RAMOS, A.; PEREIRA, F. E-readers: Fatores que influenciam a intenção de adoção. AIS Electronic Library, v. 5, 2013.

AMES, M. C. F. D. C. et al. M-commerce: analisando a influência do sexo na percepção dos usuários. Revista Gestão \& Tecnologia, v. 16, n. 2, 2016.

BEHRENS, J. The effects of familiarity and online consumer reviews, on consumers trust, risk perception, and behavioral intentions. 2014. 50 f. Dissertação (Mestrado - Behavioural, Management and Social Sciences) - University of Twente, Enschede, 2014.

BLACKWELL, R. D.; MINIARD, P. W.; ENGEL, J. F. Comportamento do consumidor. 9. ed. São Paulo: Pioneira Thomson Learning, 2005. 
CASIMIR, G.; DUTILH, C. Sustainability: a gender studies perspective. International Journal of Consumer Studies, v. 27, n. 4, p. 316-325, 2003.

CHIN, W. W. The partial least squares approach for structural equation modeling. In: MARCOULIDES, G. A. (Ed.). Modern methods for business research. London: Lawrence Erlbaum Associates, 1998, p. 295-236.

EAGLY, A. H.; DIEKMAN, A. B.; JOHANNESEN-SCHMIDT, M. C.; KOENIG, A. M. Gender gaps in sociopolitical attitudes: a social psychological analysis. Journal of Personality and Social Psychology, v. 87, n. 6, p. 796, 2004.

FERREIRA, J. P. G. E-commerce e género: análise das percepções de compra do consumidor. 2013.62 f. Dissertação (Mestrado em Marketing) - Universidade de Lisboa - Instituto Superior de Economia e Gestão, Lisboa, 2013.

FORNELL, C.; LARCKER, D. F. Evaluating structural equation models with unobservable variables and measurement error. Journal of Marketing Research, p. 39-50, 1981.

GARCIA, M. C. Escola sem fronteiras: educação superior para a sociedade digital. Revista Organicom, v. 12 , n. 23, 2015.

GOMES, M. A. da S.; SILVA, N. L. da; SILVA, A. V. Da; SADOYAMA, A. dos S. P. O comportamento do consumidor que atua no comércio eletrônico e seus reflexos na economia. Blucher Education Proceedings, v. 2, n. 1, p. 122-132, 2017.

GUTIÉRREZ, S. S. M.; LÓPEZ-CATALÁN, B.; RAMON-JERONIMO, M. A. Determinants of involvement in mobile commerce: the moderating role of gender. Esic Market Economic and Business Journal, v. 141, p. 69-10, jan. 2012.

HAIR JR J. F.; BABIN, B.; MONEY, A. H.; SAMOUEL, P. Fundamentos de métodos de pesquisa em administração. Porto Alegre: Bookman, 2005.

HAIR JR J. F.; HULT, G. T. M.; RINGLE, C.; SARSTEDT, M. A primer on partial least squares structural equation modeling (PLS-SEM). Sage Publications, 2014.

HENSELER, J.; RINGLE, C. M.; SINKOVICS, R. R. The use of partial least squares path modeling in international marketing. Advances in International Marketing (AIM), v. 20, p. 277-320, 2009.

HILL M. M.; HILL A. Investigação por questionário. Lisboa: Síbalo. 2000.

HUI, K. L.; CHAU, P. Y. K. Classifying digital products. Communications of the ACM, v. 45, n. 6, p. 73$79,2002$.

HWANG, Y.; JEONG, J. Electronic commerce and online consumer behavior research: a literature review. Information Development, v. 32, n. 3, p. 377-388, 2016.

JACIOW, M.; WOLNY, R.; STOLECKA-MAKOWSKA, A. E-consumer in Europe: comparative analysis of behaviours. Gliwice: HELION SA Publishing Group, 2013.

JONES, J. J. et al. Gender makes a difference: investigating consumer purchasing behavior and attitudes toward corporate social responsibility policies. Corporate Social Responsibility and Environmental Management, v. 24, p. 133-144, 2017.

JUNG, C. G. Os arquétipos e o inconsciente coletivo. Petrópolis: Vozes, 2000.

KARPPINEN, P. Meaningful learning with digital and online videos: theoretical perspectives. AACE Journal, v. 13, n. 3, p. 233-250, 2005.

LIMAYEM, M.; HIRT, S. G.; CHEUNG, C. M. K. How habit limits the predictive power of intention: the case of information systems continuance. MIS Quarterly, v. 31, n. 4, p. 705-737. 2007. 
LITTO, F. M. As interfaces da EAD na educação brasileira. Revista USP, n. 100, p. 57-66, 2014.

LUCHS, M. G.; MOORADIAN, T. A. Sex, personality, and sustainable consumer behaviour: elucidating the gender effect. Journal of Consumer Policy, v. 35, n. 1, p. 127-144, 2012.

LUNA, C. de J. da C. Luz, câmera, ação: os vídeos na educação em ciências e produção de saberes. 2014. 173 f. Dissertação (Mestrado em Ciências e Matemática) - Universidade Federal de Pelotas, Pelotas, 2014.

MACEDO, D. C. de; KOVALESKI, J. L.; BETIM, M. T.; MATOS, S. N. Comércio eletrônico: identificação do perfil do e-consumidor. Revista da FAE, v. 16, n. 1, p. 90-103, 2013.

MACEDO, I. M. Predicting the acceptance and use of information and communication technology by older adults: An empirical examination of the revised UTAUT2. Computers in Human Behavior, n. 75, p. 935948, 2017.

MACMILlAN, I. C.; MCGRATH, Rita G. Discover your products' hidden potential. Harvard Business Review, v. 74, n. 3, p. 58-73, 1996.

MALHOTRA, N. K. Pesquisa de marketing: uma orientação aplicada. Porto Alegre: Bookman, 2001.

MORAES, G. H. S. M. de; CAPELlOZZA, A.; MEIRELlES, F. de S. A tecnologia da informação e os movimentos sociais: um estudo do movimento Passe Livre. Gestão \& Regionalidade, v. 33, n. 97, jan./abr. 2017.

MARASSI, A. de C. B. Consumo do livro no ciberespaço: e-commerce e redes sociais. e-Com, v. 6, n. 1, 2013.

MOREIRA, R. A. O Comércio eletrônico, os métodos de pagamentos e os mecanismos de segurança. RefasRevista Fatec Zona Sul, v. 3, n. 1, p. 16-30, 2016.

MORGADO, M. G. Comportamento do consumidor online: perfil, uso da internet e atitudes. $2003.160 \mathrm{f}$. Tese (Doutorado em Administração) - FGV - EAESP, São Paulo, 2003.

OKAZAKI, S.; MENDEZ, F. Exploring convenience in mobile commerce: moderating effects of gender. Computers in Human Behavior, v. 29, n. 3, p. 1234-1242, 2013.

RINGLE, C. M.; DA SILVA, D.; BIDO, D. de S. Modelagem de equações estruturais com utilização do SmartPLS. Revista Brasileira de Marketing, v. 13, n. 2, p. 56-73, 2014.

RIQUELME, H. E.; RIOS, R. E. The moderating effect of gender in the adoption of mobile banking. International Journal of Bank Marketing, v. 28, n. 5, p. 328-341, 2010.

SILVEIRA, A. B. da. Atitudes e intenções de adoção de internet móvel: uma análise do comportamento do consumidor jovem adulto. 2012184 f. Dissertação (Mestrado em Administração e Negócios) - Pontifícia Universidade Católica do Rio Grande do Sul, Porto Alegre, 2012.

SOLOMON, M. R. O comportamento do consumidor: comprando, possuindo e sendo. 11. ed. Porto Alegre: Bookman Editora, 2016.

SORDI, J. O.; NELSON, R. E.; MEIRELES, M.; SILVEIRA, M. A. da. Development of digital products and services: proposal of a framework to analyze versioning actions. European Management Journal, n. 34, p. 564-578, 2016.

TORI, R. Tecnologia e metodologia para uma educação sem distância. EaD EmRede-Revista de Educação a Distância, v. 2, n. 2, p. 44-55, 2015.

TORRES, A. Pós-modernidade e consumo: desafios ao neo-marketing. European Journal of Applied Business and Management, v. 2, n. 1, 2015. 
VENKATESH, V.; MORRIS, M. G., DAVIS, G. B.; DAVIS, F. D. User acceptance of information technology: toward a unified view. MIS Quarterly, v. 27, n. 3, p. 425-478, 2003.

VENKATESH, V.; THONG, J. Y. L.; XU, X. Consumer acceptance and use of information technology: extending the unified theory of acceptance and use of technology. MIS Quarterly, v. 36, n. 1, p. 157-178, mar. 2012.

XU, H. M-commerce development in China: users' perspectives. Journal of Technology Research. vol. 5, 2014.

\section{APÊNDICE A - Relação dos construtos e assertivas utilizadas no questionário}

\begin{tabular}{|c|c|c|}
\hline Fatores & Construtos & Assertivas \\
\hline 01 - Segurança & $\begin{array}{l}\text { Quanto o } \text { consumidor } \\
\text { acredita estar realizando } \\
\text { um procedimento seguro na } \\
\text { aquisição de um produto } \\
\text { digital. }\end{array}$ & $\begin{array}{l}\text { Seg1 - Penso que se deve comprar na Internet apenas se tiver } \\
\text { segurança no site que está realizando a compra. } \\
\text { Seg2 - Sempre que vou realizar uma compra na internet, } \\
\text { verifico se o site é seguro, se possui certificados de segurança } \\
\text { e apresenta outras informações relativas à segurança. } \\
\text { Seg3 - Encontrando o produto ideal para comprar na internet, } \\
\text { só realizo a compra se verificar que o site é seguro. }\end{array}$ \\
\hline $\begin{array}{l}02-\text { Expectativa de } \\
\text { desempenho }\end{array}$ & \begin{tabular}{lcrr} 
Quanto & o & \multicolumn{2}{c}{ consumidor } \\
acredita & que & o uso do \\
produto & o & ajudará a \\
alcançar & o & benefício \\
almejado. & & &
\end{tabular} & $\begin{array}{l}\text { ED1 - Penso que produtos digitais educacionais são úteis na } \\
\text { minha vida. } \\
\text { ED2 - Acredito que posso aprender utilizando produtos } \\
\text { digitais educacionais. } \\
\text { ED3 - Utilizar produtos digitais educacionais pode me ajudar } \\
\text { a conquistar meus objetivos em relação à educação. } \\
\text { ED4 - Penso que posso adquirir conhecimento utilizando um } \\
\text { produto digital educacional. }\end{array}$ \\
\hline $\begin{array}{l}03 \text { - Expectativa de } \\
\text { esforço }\end{array}$ & $\begin{array}{l}\text { Quanto o consumidor } \\
\text { acredita que irá dispender } \\
\text { de esforço para utilizar o } \\
\text { produto. }\end{array}$ & $\begin{array}{l}\text { EE1 - Tenho facilidade em usar a internet. } \\
\text { EE2 - Usar livros eletrônicos (e-books) na internet é fácil. } \\
\text { EE3 - Usar vídeos na internet é fácil. } \\
\text { EE4 - Ler livros eletrônicos (e-books) é fácil. } \\
\text { EE5 - Assistir a vídeos na internet é fácil. }\end{array}$ \\
\hline 04 - Influência social & $\begin{array}{l}\text { Quanto o consumidor } \\
\text { percebe de importância da } \\
\text { opinião dos outros na } \\
\text { avaliação do produto. }\end{array}$ & $\begin{array}{l}\text { IS1 - Uso recomendações e comentários on-line de outras } \\
\text { pessoas para obter informações sobre produtos digitais } \\
\text { educacionais. } \\
\text { IS2 - Creio que as recomendações e comentários on-line } \\
\text { sobre produtos educacionais são verdadeiros. } \\
\text { IS3 - As recomendações e comentários on-line me permitem } \\
\text { obter produtos educacionais adequados para minhas } \\
\text { necessidades. } \\
\text { IS4 - Comentários de outras pessoas, que estejam junto ao } \\
\text { produto digital que pretendo comprar, me ajudam na decisão } \\
\text { de compra. }\end{array}$ \\
\hline $05 \quad-\quad$ Condições & $\begin{array}{lrr}\text { Quanto } & \text { o } & \text { consumidor } \\
\text { acredita } & \text { na } & \text { infraestrutura }\end{array}$ & CF1 - Tenho os meios necessários para realizar uma compra \\
\hline
\end{tabular}




\begin{tabular}{|c|c|c|}
\hline facilitadoras & $\begin{array}{l}\text { organizacional e técnica } \\
\text { que dá suporte ao uso do } \\
\text { produto. }\end{array}$ & $\begin{array}{l}\text { on-line. } \\
\text { CF2 - Tenho os meios (computador, tablet etc.) adequados } \\
\text { para utilizar um livro eletrônico (e-book) ou um vídeo na } \\
\text { internet. } \\
\text { CF3 - Consigo facilmente acessar o conteúdo de estudos on- } \\
\text { line, sempre que preciso. }\end{array}$ \\
\hline $\begin{array}{ll}06 \quad \text { - } & \text { Motivação } \\
\text { hedônica } & \end{array}$ & $\begin{array}{llr}\text { Quanto } & \text { o consumidor } \\
\text { percebe o uso do produto } \\
\text { sendo divertido } \\
\text { prazeroso. }\end{array}$ & $\begin{array}{l}\text { MH1 - Estudar utilizando um livro eletrônico (e-book) é } \\
\text { prazeroso. } \\
\text { MH2 - Estudar utilizando um vídeo na internet é prazeroso. } \\
\text { MH3 - O uso de um livro eletrônico é agradável. } \\
\text { MH4 - O uso de um vídeo na internet é agradável. }\end{array}$ \\
\hline 07 - Preço & $\begin{array}{l}\text { Quanto o valor monetário } \\
\text { irá influenciar na adoção do } \\
\text { produto. }\end{array}$ & $\begin{array}{l}\text { Pre1 - Cursos (que utilizam livros eletrônicos e vídeos) } \\
\text { vendidos na Internet têm um preço razoável. } \\
\text { Pre } 2 \text { - Os cursos disponíveis na Internet valem o preço que é } \\
\text { cobrado. } \\
\text { Pre3 - Tendo em vista o preço que hoje é cobrado, os livros } \\
\text { eletrônicos (e-books) e vídeos educacionais disponíveis pela } \\
\text { Internet têm um valor adequado. } \\
\text { Pre4 - Só uso livros e vídeos pela Internet de forma gratuita. }\end{array}$ \\
\hline 08 - Hábito & $\begin{array}{l}\text { Quanto o consumidor já } \\
\text { teve experiência com } \\
\text { elementos correlatos ao do } \\
\text { produto. }\end{array}$ & $\begin{array}{l}\text { Ha1 - Utilizar produtos educacionais digitais é um hábito para } \\
\text { mim. } \\
\text { Ha2 - Costumo estudar utilizando livros eletrônicos (e- } \\
\text { books). } \\
\text { Ha3 - Estou acostumado a usar vídeos na internet para } \\
\text { estudar. }\end{array}$ \\
\hline 09 - Intenção de Uso & \begin{tabular}{lcr} 
De que & forma & o \\
consumidor & irá & se \\
comportar, & se pretende \\
utilizar & determinado \\
produto. & \multicolumn{2}{c}{}
\end{tabular} & $\begin{array}{l}\text { IU1 - Pretendo usar produtos digitais educacionais no futuro. } \\
\text { IU2 - Tentarei usar produtos digitais educacionais em minha } \\
\text { rotina. } \\
\text { IU3 - Planejo utilizar produtos digitais educacionais com } \\
\text { frequência. } \\
\text { IU4 - Darei preferência ao aprendizado por meio de produtos } \\
\text { digitais educacionais. }\end{array}$ \\
\hline
\end{tabular}

